\title{
A phase I multicenter study of antroquinonol in patients with metastatic non-small-cell lung cancer who have received at least two prior systemic treatment regimens, including one platinum-based chemotherapy regimen
}

\author{
YU-CHIN LEE ${ }^{1,2}$, CHING-LIANG HO ${ }^{3}$, WOEI-YAU KAO ${ }^{4}$ and YUH-MIN $\mathrm{CHEN}^{2,5,6}$ \\ ${ }^{1}$ Sijhih Cathay General Hospital, New Taipei 221; ${ }^{2}$ School of Medicine, National Yang-Ming University, Taipei 112; \\ ${ }^{3}$ Tri-Service General Hospital, National Defense Medical Center, Taipei 114; ${ }^{4}$ Taipei Tzu Chi General Hospital, \\ Taipei 231; ${ }^{5}$ Department of Chest Medicine, Taipei Veterans General Hospital, Taipei 11217; ${ }^{6}$ College of \\ Medical Science and Technology, Taipei Medical University, Taipei 110, Taiwan, R.O.C.
}

Received March 27, 2015; Accepted September 8, 2015

DOI: $10.3892 / \operatorname{mco} .2015 .642$

\begin{abstract}
Antroquinonol is isolated from Antrodia camphorata, a camphor tree mushroom, and is a valuable traditional Chinese herbal medicine that exhibits pharmacological activities against several diseases, including cancer. This first-in-human phase I study of antroquinonol included patients with metastatic non-small-cell lung cancer who had received at least two prior systemic treatment regimens. An open-label, dose escalation, pharmacokinetic (PK) study was conducted to determine the maximum tolerable dose (MTD), dose-limiting toxicities (DLTs), and safety/tolerability and preliminary efficacy profiles of antroquinonol. The patients received escalating doses of once-daily antroquinonol in 4-week cycles (up to 3 cycles). The escalated doses were 50-600 mg. PKs were evaluated on day 1 and 28 of cycle 1 . Between January, 2011 and October, 2012, 13 patients with metastatic adenocarcinoma were enrolled. No DLTs occurred in any patient at any dose level. $\mathrm{T}_{\max }$ was observed between 1.00 and $3.70 \mathrm{~h}$ under single-dose conditions, and at 1.92-4.05 h under multiple-dose conditions. The mean elimination half-life ranged between 1.30 and $4.33 \mathrm{~h}$, independent of the treatment dose. Antroquinonol at all dose levels had a mild toxicity profile, with no reported treatment-related mortality. The most common treatment-related adverse events were diarrhea, vomiting and nausea. The best tumor response was stable disease in 3 patients. In conclusion, antroquinonol at all dose levels, administered daily for 4 weeks, was generally safe and
\end{abstract}

Correspondence to: Professor Yuh-Min Chen, Department of Chest Medicine, Taipei Veterans General Hospital, Sec. 2, 201 Shihpai Road, Taipei 11217, Taiwan, R.O.C.

E-mail: ymchen@vghtpe.gov.tw

Key words: Antrodia camphorata, antroquinonol, phase I, lung cancer well tolerated, without DLTs. The recommended dose level for a phase II study is $\geq 600 \mathrm{mg}$ daily.

\section{Introduction}

Lung cancer is the leading cause of cancer-related mortality worldwide. Over $85 \%$ of lung cancers are non-small-cell lung cancers (NSCLCs), with adenocarcinoma being the most common histological subtype of NSCLC. Despite continuous advances in the diagnosis and treatment of NSCLC, 90\% of newly diagnosed patients with advanced NSCLC succumb to the disease within 2 years. As the rate of disseminated disease in such patients is high, numerous attempts have been made to improve systemic treatment for over 2 decades (1).

Antrodia camphorata, a camphor tree mushroom, is a valuable traditional Chinese herbal medicine that exhibits pharmacological properties against several diseases. Antrodia camphorata is rich in flavonoids, terpenoids, polyphenolics and polysaccharides, and has been produced on an agricultural manufacturing scale in Taiwan. The alcohol extract of Antrodia camphorata had been found to exert anticancer effects through inhibiting tumor cell DNA synthesis, promoting apoptosis and exerting an antimigration effect (2).

Antroquinonol is a ubiquinone derivative isolated from Antrodia camphorata. A previous study demonstrated that antroquinonol displayed anticancer activity against hepatocellular carcinoma cell lines through activation of 5 ' adenosine-monophosphate-activated protein kinase and inhibition of the mammalian target of rapamycin (mTOR) pathway (3). Further study demonstrated that antroquinonol exhibits anticancer activity in human pancreatic cancers through inhibition of the phosphoinositide-3 kinase (PI3K)/Akt/mTOR pathway, which in turn downregulates the expression of cell cycle regulators (4). The translational inhibition causes a G1 arrest of the cell cycle and ultimately mitochondria-dependent apoptosis. Moreover, autophagic cell death and accelerated senescence may also explain the antroquinonol-mediated anticancer effect. A study on the 
A549 pulmonary adenocarcinoma cell line demonstrated that antroquinonol-induced apoptosis was associated with disrupted mitochondrial membrane potential and activation of caspase-3 and poly ADP ribose polymerase cleavage (5). Moreover, antroquinonol treatment downregulated the expression of B-cell lymphoma 2 proteins, which was correlated with decreased PI3K and mTOR protein levels, without altering the levels of pro- or antiapoptotic proteins. The results of the microarray analysis demonstrated that antroquinonol altered the expression level of miRNAs in A549 cells compared with the untreated control. These data collectively suggested that antroquinonol exerted an antiproliferative effect on A549 cells (5). In addition, antroquinonol exhibited no observed genotoxicity or reproductive toxicity at doses up to $80 \mathrm{mg} / \mathrm{kg} /$ day in the Ames test, mammalian cell gene mutation test, micronucleus test, erythrocyte micronucleus test, or in vivo study of Sprague Dawley rats (unpublished data).

Thus, this multicenter phase I trial was conducted to determine the maximum tolerable dose (MTD) and evaluate the pharmacokinetics (PKs), safety, tolerability and efficacy profiles of antroquinonol use in metastatic NSCLC patients who had received at least two prior systemic treatment regimens, including one platinum-based chemotherapy regimen.

\section{Patients and methods}

Study design and treatment. This was an open-label, non-randomized, dose escalation PKs study conducted to determine the MTD and dose-limiting toxicities (DLTs) and investigate the PKs, safety, tolerability and preliminary efficacy profiles of antroquinonol. An accelerated titration design was used for this study. For the 6 dose levels (daily dose of 50,100, 200, 300, 450 and $600 \mathrm{mg}$ ), a maximum of 36 patients were scheduled based on the criteria of a maximum of 6 patients per cohort: 1 (lowest level) to 6 (highest level) patients were scheduled for each dose group in the accelerated titration phase, and 3-6 patients for each dose group in the standard titration phase (6 dose cohorts) (Fig. 1). Antroquinonol was administered per os daily, within 15 min after breakfast at the assigned dose levels for 4 weeks. The study protocol was approved by the Institutional Review Boards of Taipei Veterans General Hospital and Tri-Service General Hospital (VGHIRB 201010007MB, TSGHIRB 099-01-007, clinicaltrials.gov protocol registration system NCT011-340-16).

The primary endpoint was MTD. DLT was defined as any $\geq$ grade 3 toxicity according to the National Cancer Institute Common Terminology Criteria for Adverse Events (NCI CTCAE), version 4.03 (http://evs.nci.nih.gov/ftp1/ CTCAE/CTCAE_4.03_2010-06-14_QuickReference_5x7.pdf), as determined by the investigator, possibly related in terms of causality to the administration of the study drug, and observed during the first 4 weeks of treatment for patients in the accelerated or standard titration phase. Grade $\geq 3$ nausea, vomiting and diarrhea were considered as DLTs only if they remained at grade 3 for $>3$ days despite adequate treatment. The secondary endpoints included three parameters: PKs, preliminary efficacy evaluation of changes in measurable tumor size, and safety profile.

No other cytotoxic agents, targeted therapy, investigational drugs, immunotherapy, or radiotherapy was permitted during

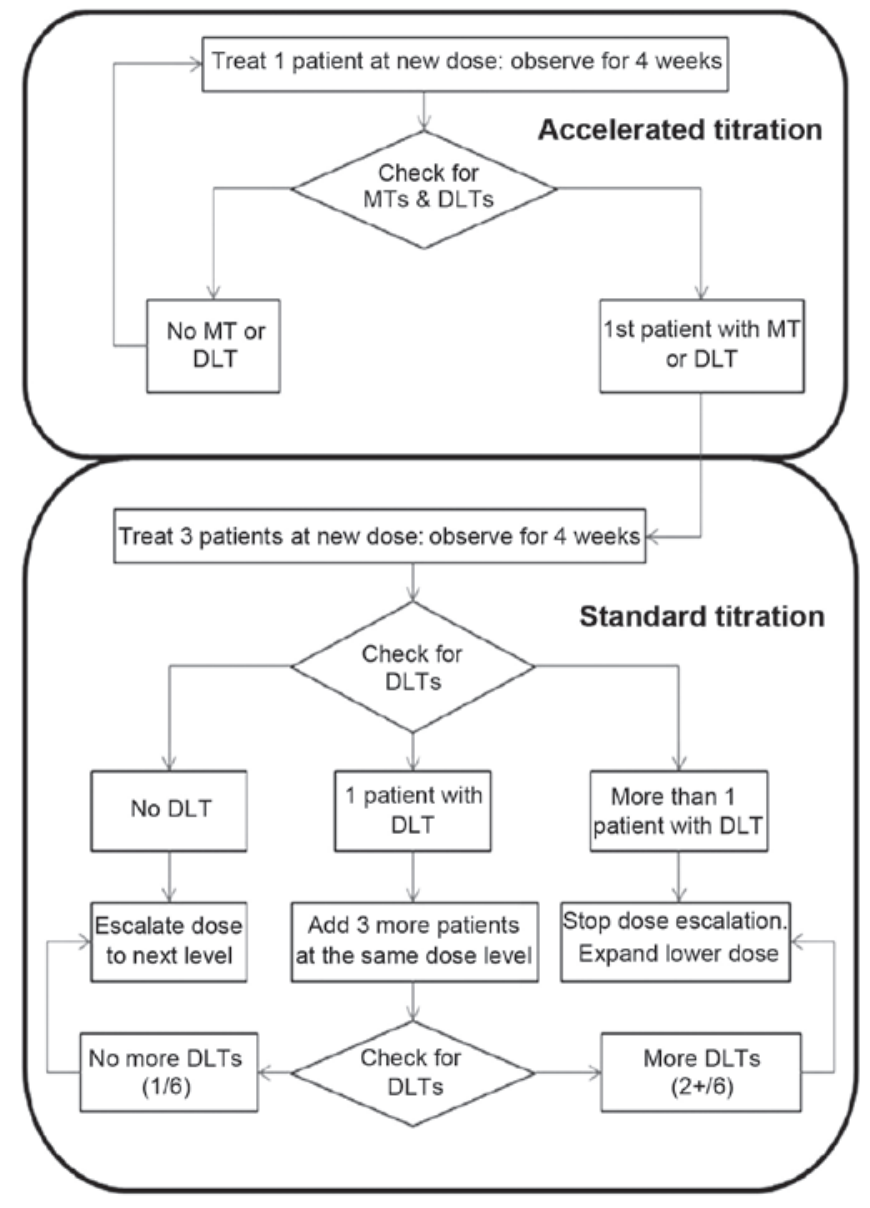

Figure 1. Dose titration schematic diagram. An accelerated titration design was used for this study. The method of dose escalation in the accelerated titration phase was to continue to the next higher dose level until a patient experienced moderate toxicity (MT) or any dose-limiting toxicity (DLT). When either occurred, the accelerated titration phase was terminated. Any DLT or MT during any 4-week treatment at any dose level led to the initiation of the standard titration $(3+3)$ phase. During this phase, the patients were enrolled in a cohort of 3 patients per dose level and treated for 4 weeks at that level. A cycle of treatment during the standard titration phase was defined as 4 weeks of treatment. Dose escalation proceeded sequentially between cohorts. An internal cohort review committee monitored the study on an ongoing basis to determine if the treatment could be taken to the next dose level.

the study period. The following medications were also prohibited: Levonorgestrel (internal standard in bioanalysis), and drugs known to inhibit or induce cytochrome P (CYP)2C19, CYP3A4, CYP2C8 and CYP2E1.

Patients. Patients aged $\geq 20$ years with metastatic NSCLC of the lung who had developed progressive disease after two lines of chemotherapy (including one platinum-based) and one line of epidermal growth factor receptor (EGFR)-targeted therapy (if the patient harboured an EGFR mutation or if the EGFR mutation status was unknown) were enrolled in this study, after providing written informed consent. Other eligibility criteria included: A histological or cytological diagnosis of adenocarcinoma of the lung (or mixed-cell type tumor with an adenocarcinomatous component); life expectancy of $\geq 3$ months; Eastern Cooperative Oncology Group performance status score of $\leq 2$; recovery from toxicities of 
previous anticancer treatments to $\leq$ grade 1 NCI CTCAE, except for alopecia; clinically measurable disease; no previous radiotherapy directed at the measurable lesion(s); adequate bone marrow reserve, with a white blood cell count of $\geq 3,500 / \mathrm{mm}^{3}$, hemoglobin concentration of $\geq 9.0 \mathrm{~g} / \mathrm{dl}$, and platelet count of $\geq 100,000$ cells $/ \mathrm{mm}^{3}$; adequate liver function; and adequate renal function.

The patients were categorized into an intent-to-treat (ITT) population (patients who received at least one dose of antroquinonol), a per-protocol (PP) population [patients who completed at least 3 cycles of treatment with proper imaging assessment according to Response Evaluation Criteria in Solid Tumors (RECIST) (6)] and a PK population (patients who received at least one dose of antroquinonol with sufficient post-dose bio-samples collected for PKs profile characterization). Baseline and demographic characteristics and safety analyses were performed in the ITT population. The PK population was used for PKs analyses.

PKs evaluation. For each dose cohort, samples were obtained from all patients treated in the accelerated and standard titration phases of the study. Blood sampling was performed on days 1, 14, 27 and 28 of the first treatment cycle only. Serial blood samples ( $5 \mathrm{ml}$ per sample) were collected for $24 \mathrm{~h}$ at the following time points on study days 1 and 28: Day 1, within $30 \mathrm{~min}$ prior to and at $0.25,0.5,1,2,3,4,6,8,10,14$ and $24 \mathrm{~h}$ after dose administration; and day 28 , immediately prior to and at $0.25,0.5,1,2,3,4,6,8,10,14$ and $24 \mathrm{~h}$ after dose administration. A blood sample for trough plasma concentration was collected immediately prior to dose administration on days 14 and 27.

Determining the plasma PKs of antroquinonol involved investigating the following parameters during a maximum of 4 weeks of dose administration in the accelerated and standard titration phases: $\mathrm{C}_{\max \text { day }}$, observed maximum plasma concentration after dosing on day $1 ; \mathrm{T}_{\max \text { day } 1}$, time to reach $\mathrm{C}_{\text {max day } 1}$ on day $1 ; \mathrm{AUC}_{0-\mathrm{t} \text { day } 1}$, truncated area under the plasma concentration-time curve from the beginning of dosing to the last measurable concentration on day $1 ; \mathrm{T}_{1 / 2 \text { dayl }}$, half-life on day $1 ; \mathrm{C}_{\max \text { day } 28}$, observed maximum plasma concentration after dosing on day $28 ; \mathrm{T}_{\max \text { day } 28}$, time to reach $\mathrm{C}_{\max \text { day } 28}$ on day 28; $\mathrm{AUC}_{0-\mathrm{t} \mathrm{day} 28}$, truncated area under the plasma concentration-time curve from the beginning of dosing to the last measurable concentration on day $28 ; \mathrm{T}_{1 / 2 \text { day } 28}$, half-life on day 28; $\mathrm{T}_{1 / 2 \text { effective }}$, half-life derived from the observed antroquinonol accumulation; $\mathrm{C}_{\text {trough day14}}$, trough plasma concentration on day $14 ; \mathrm{C}_{\text {trough day } 27}$, trough plasma concentration on day $27 ; \mathrm{C}_{\text {trough day } 28}$, trough plasma concentration on day 28 .

Safety and efficacy assessments. The tumor burden was measured according to RECIST, version 1.1, at baseline and at treatment discontinuation. Safety evaluation included complete blood cell counts, biochemistry laboratory data, C-reactive protein level, urinalysis, vital signs, electrocardiogram (ECG) examination and adverse events (AEs).

Patients in the accelerated titration phase were treated for 4 weeks at their first dose level, and then continued with treatment in the extension program for 3 cycles if found to be eligible. Moderate toxicity (MT) and DLTs were observed during the initial 4-week treatment period. The accelerated phase ended when either a DLT or MT was observed. A patient who experienced a DLT at any other level besides the lowest level was returned to the previous dose level. A patient who experienced a DLT at the lowest dose level was discontinued from the study. Patients in the standard titration phase were treated with antroquinonol for 4 weeks. DLTs were evaluated during the 4-week treatment period.

Prior to each 4-week cycle and at mid-cycle (i.e., day 14), a blood sample was drawn for complete blood testing in order to determine whether it was safe to continue with the study drug. If there was a $\geq$ grade 3 toxicity, the patient's dose was reduced to the next lower dose. Dose escalation was allowed in the study and each patient was allowed two dose escalations. Patients who required a dose reduction due to toxicity were not allowed dose escalation at any point during the study. The patients were observed for AEs until 6 weeks after their last treatment.

Extension program. The extension program included patients who had not withdrawn from the study and wished to participate in the accelerated or standard titration phase following their treatment cycle. The patients were treated for up to three 4-week cycles for as long as they were willing to continue, or until development of intolerable toxicity or progressive disease.

Statistical analysis. The PKs variables were calculated from the serum concentration data using standard, non-compartmental methods as implemented in WinNonlin, version 5.2 or higher (Pharsight Corp., St. Louis, MO, USA). PKs parameters were presented with descriptive statistics by dose level. Dose proportionality was to be evaluated if sufficient data were available in dose groups.

All statistical analyses were performed using SAS software, version 8.2 or higher (SAS Institute Inc. Cary, NC, USA). Categorical data were summarized using counts and percentages. For continuous variables, descriptive statistics were tabulated, such as number of available observations, mean, median, standard deviation, minimum and maximum. All available data and the tabulation of the results were displayed by initial dose level and with all levels pooled as a whole if applicable. All statistical tests were two-sided, with a P-value of $<0.05$ indicating statistically significant differences.

\section{Results}

Patients. A total of 13 patients ( 7 men and 6 women) were enrolled in this study. All the patients were ethnic Chinese and their mean age was 61 years (range, $43-86$ years). Two patients harboured EGFR-activating mutations, 4 patients had EGFR wild-type and 7 patients had unknown EGFR status.

A total of 5 patients were enrolled in the accelerated titration phase (1 patient each in the 50-, 100-, 200-, 300- and 450-mg dose groups) and 8 patients in the standard titration phase (3 patients in the 450- and 5 patients in the 600-mg dose group). All 13 patients were included in the ITT and the PK populations. The PP population consisted of 3 patients, with 1 patient in the 200 - and 2 patients in the 600 -mg dose group.

DLT and MTD. There were no DLTs reported in any patient for any of the dose levels in the ITT population in the accelerated 


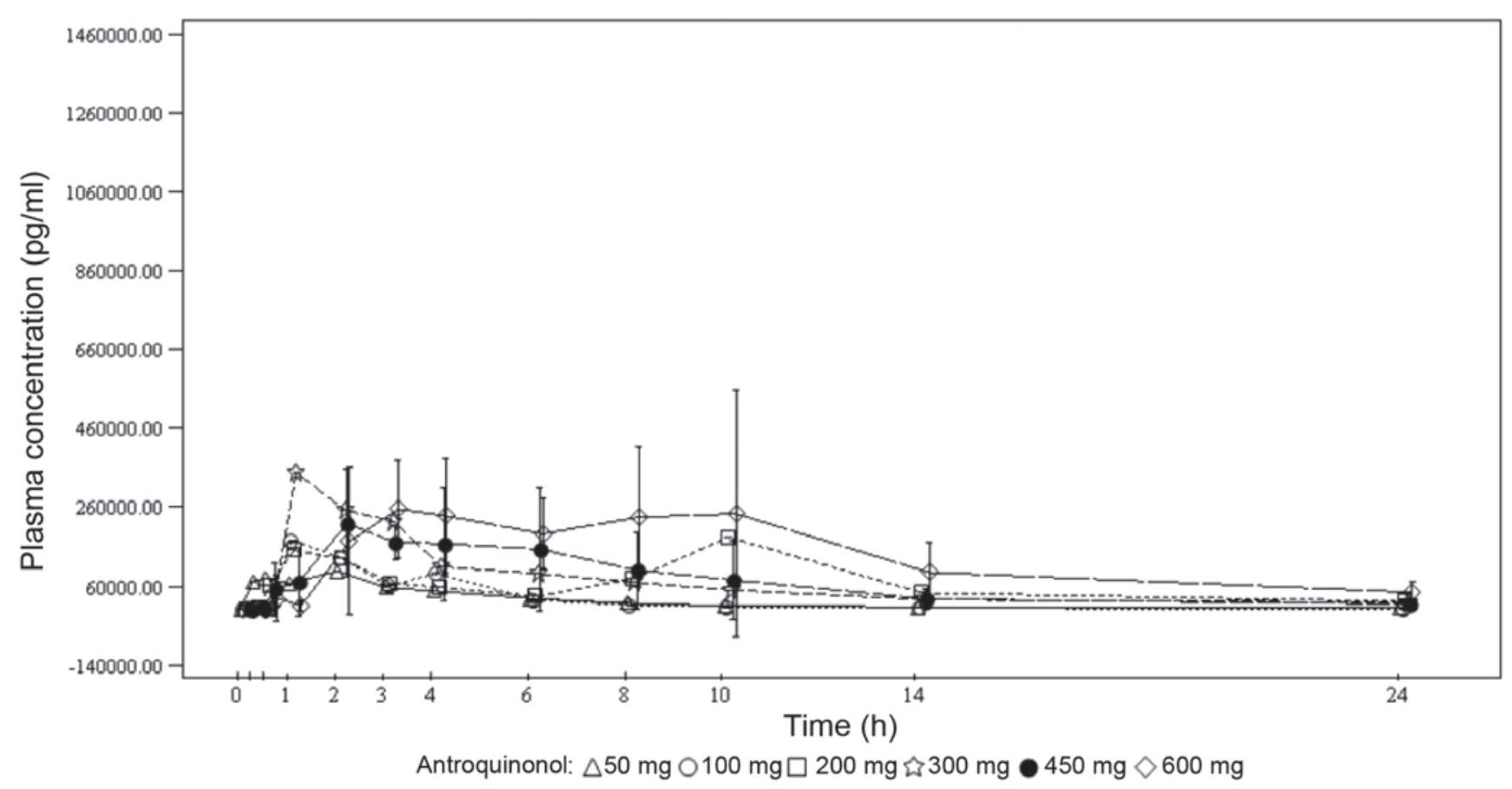

Figure 2. Pharmacokinetics (PKs) mean plasma concentrations ( \pm standard deviation) vs. time by treatment dose (original scale), PK population - day 1.

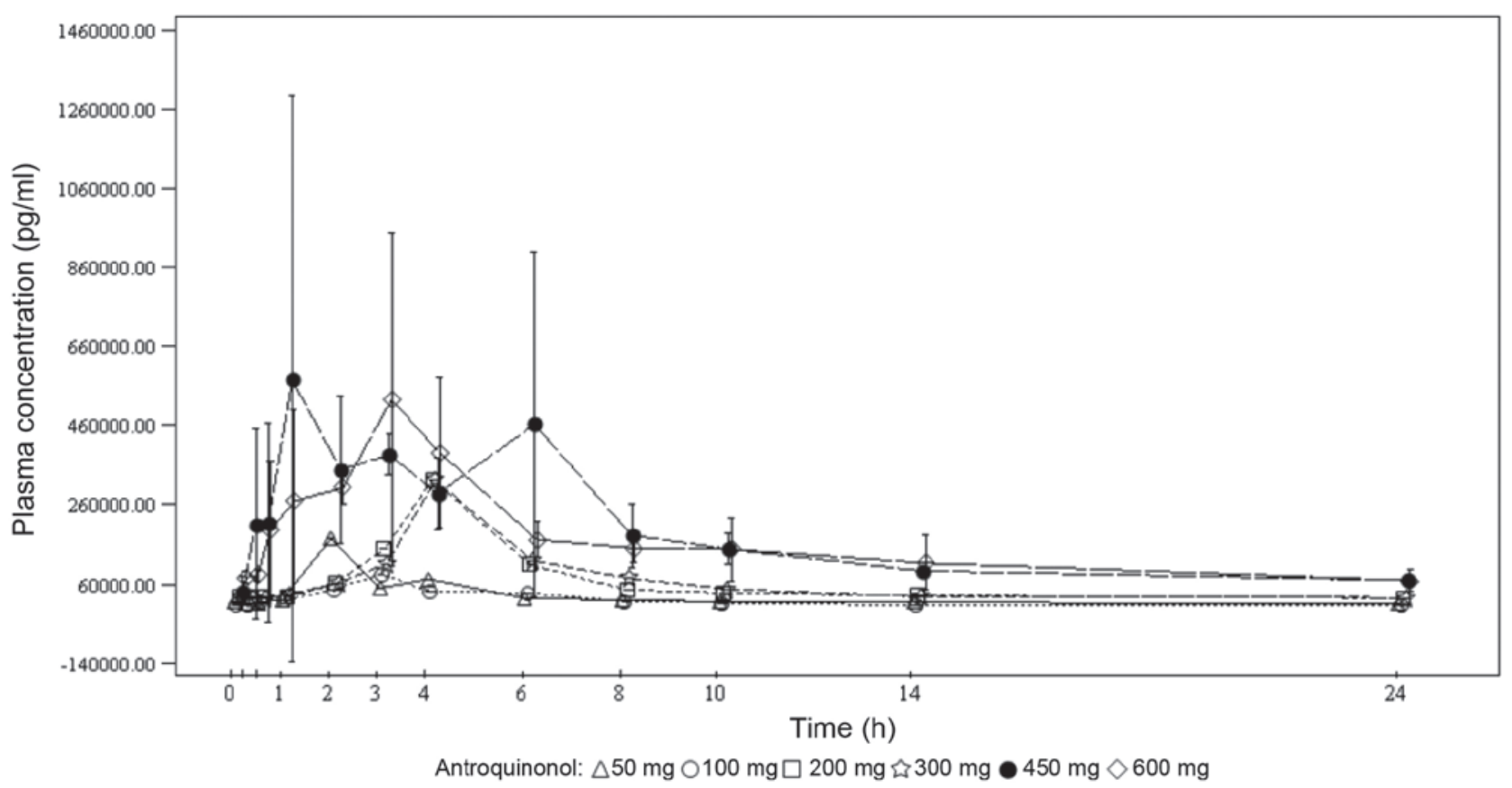

Figure 3. Pharmacokinetics (PKs) mean plasma concentrations ( \pm standard deviation) vs. time by treatment dose (original scale). PK population - day 28.

or standard titration phase. An MTD was not determined in this study, and was $\geq 600 \mathrm{mg}$ daily.

Efficacy. Four patients continued to the fourth treatment cycle, 1 patient each in the 200- and 450-mg dose groups and 2 patients in the $600-\mathrm{mg}$ dose group. The best overall response was stable disease in 3 patients (1 patient in the 200 -mg dose group and 2 patients in the 600-mg dose group), progressive disease in 7 patients, and not evaluated in 3 patients. Two patients were identified with EGFR-activating mutations, including 1 patient in the 450-mg dose group who discontinued the study drug prior to the completion of the first cycle and 1 patient in the 600-mg dose group who completed two cycles of treatment with the minor response of the sum of the longest diameters of the target lesions being reduced from $41 \mathrm{~mm}$ at screening to $31 \mathrm{~mm}$ at the end of the study visits.

Safety. Overall, antroquinonol at all dose levels exhibited a mild toxicity profile. A total of 4 patients reported 4 serious AEs (2 patients each in the 450- and 600-mg dose groups, 
including 1 patient from each dose group who succumbed to progressive disease). None of the mortalities, serious AEs or AEs leading to discontinuation was related to the study drug. No grade 4 treatment-emergent AEs (TEAEs) were reported. Grade 3 TEAEs were reported in 2 patients ( 1 patient in the 200-mg dose group experienced vertigo and 1 patient in the 450-mg dose group experienced decreased appetite). The most commonly occurring TEAEs reported in $>2$ patients were diarrhea [10 patients, $76.9 \%$ (grade 1, 9 patients; and grade 2, 1 patient)], vomiting [9 patients, $69.2 \%$ (grade 1,6 patients; and grade 2, 3 patients)], and nausea [7 patients, $53.8 \%$ (grade 1 , 6 patients; and grade 2, 1 patient)]. No patient exhibited any laboratory abnormalities recorded as TEAEs, apart from 1 case of grade 1 hematuria in the 450-mg dose group. There were mild increases or decreases in the hematological and biochemical parameters, but none were considered as abnormal results. None of the patients suffered from clinically significant changes in any of the vital signs, ECG readings, or physical examination parameters.

$P K$ analysis. Due to the limited number of patients per dose group (1 patient each received 50, 100, 200 and $300 \mathrm{mg}$, 4 patients received $450 \mathrm{mg}$, and 5 patients received $600 \mathrm{mg}$ ), the PK results should be interpreted with caution. The power model approach was used to assess the dose proportionality of several antroquinonol doses, ranging from 50 to $600 \mathrm{mg}$. The values for slope (95\% confidence interval) for area under the curve (AUC) $)_{0-t}$ and $\mathrm{C}_{\max }$ for day 1 were $0.641(0.16-1.12)$ and 0.504 (0.17-0.83), respectively, and for day 28 they were $0.957(0.59-1.32)$ and $0.682(0.08-1.28)$, respectively. These results indicate a dose-proportional increase over the dose range for $\mathrm{AUC}_{0-\mathrm{t}}$ but not for $\mathrm{C}_{\max }$ under single-dose conditions, and a dose-proportional increase over the dose range for both $\mathrm{AUC}_{0-\mathrm{t}}$ and $\mathrm{C}_{\text {max }}$ under multiple-dose conditions.

Under single-dose conditions, the maximum antroquinonol concentration was generally observed between 1.00 and $3.70 \mathrm{~h}$, with the exception of the 200-mg dose group, where a median $\mathrm{T}_{\max }$ of $10.00 \mathrm{~h}$ was observed. Under multiple-dose conditions, the results were similar with the median $\mathrm{T}_{\max }$ ranging from 1.92 to $4.05 \mathrm{~h}$. Antroquinonol was rapidly eliminated following single and multiple administrations, with a mean $\mathrm{T}_{1 / 2}$ ranging from 1.30 to $4.33 \mathrm{~h}$, independently of the treatment dose. The trough plasma concentrations were similar on days 14, 27 and 28 within each dose group, and increased with the treatment dose. These results suggest that the steady state was probably attained on day 14 . In terms of accumulation, no clear trend was observed when comparing the rate and extent of absorption on day 1 to those on day 28. The PKs mean plasma concentrations vs. time by treatment dose on days 1 and 28 are presented in Figs. 2 and 3, respectively.

\section{Discussion}

Antrodia camphorata is an endemic species in Taiwan and has been traditionally used for food and drug intoxication, and for the treatment of diarrhea, abdominal pain, hypertension, pruritus and liver cancer (7). Other studies have reported Antrodia camphorata to possess detoxifying, anti-inflammatory, immunomodulatory, hepatoprotective and anticancer properties (8-11).
Extracts of Antrodia camphorata have been used in China as an alternative anticancer agent for decades. Previous in vitro studies have demonstrated that antroquinonol displays anticancer activity against several cancer cell lines, including the A549 pulmonary adenocarcinoma cell line (3-5). The present first-in-human study further investigated the DLT and MTD of antroquinonol and its recommended dose for phase II studies in different types of cancer.

Antroquinonol at all dose levels, administered daily for 4 weeks, was generally found to be safe and tolerable. The AEs were mainly gastrointestinal, including diarrhea, vomiting and nausea. Since no DLTs occurred in this study, the MTD could not be determined. The overall response was stable disease in 3 patients and progressive disease in 7 patients. Among the 5 patients in the $600-\mathrm{mg}$ dose level, 3 patients were evaluable for treatment response and 2 achieved stable disease for $>3$ months. However, these data should be interpreted with caution due to the limited number of patients in this study.

It may have been preferable to perform tissue biopsy and/or serum biomarker examination during antroquinonol treatment to document its effects on signal transduction pathways or tumor cell cycles when the patients were under different dose levels. However, this part was omitted, as only few patients with different dose levels of treatment were expected to be evaluable and it was difficult to persuade patients to undergo another tissue biopsy or further blood tests for biomarker studies. In addition, biomarker studies, including tissue sample studies that will be performed in the phase II study, would be more appropriate, as higher patient numbers and more biomarker findings may be correlated with treatment efficacy.

In conclusion, antroquinonol administered up to a dose level of $600 \mathrm{mg}$ daily for 4 weeks was generally safe and tolerable, as no particular safety concerns or DLTs were identified in the present study. Progression of antroquinonol to further clinical development is required. The data generated from the safety and PK profiles in this study support further studies of antroquinonol in a larger number of cancer patients. The recommended phase II dose is at least $600 \mathrm{mg}$ daily, since DLTs did not occur in the present study.

\section{Acknowledgements}

The authors would like to thank the participating patients and their families, as well as Dr Howard Cheng (Clinical Research R\&D, Golden Biotechnology Corp., New Taipei, Taiwan) for his contribution to this study.

\section{References}

1. Pfister DG, Johnson DH, Azzoli CG, et al: American Society of Clinical Oncology treatment of unresectable non-small-cell lung cancer guideline: update 2003. J Clin Oncol 22: 330-353, 2004.

2. Chiou JF, Wu AT, Wang WT, Kuo TH, Gelovani JG, Lin IH, Wu CH, Chiu WT and Deng WP: A preclinical evaluation of Antrodia camphorata alcohol extracts in the treatment of non-small cell lung cancer using non-invasive molecular imaging. Evid Based Complement Alternat Med 2011: 914561, 2011.

3. Chiang PC, Lin SC, Pan SL, Kuo CH, Tsai IL, Kuo MT, Wen WC, Chen P and Guh JH: Antroquinonol displays anticancer potential against human hepatocellular carcinoma cells: a crucial role of AMPK and mTOR pathways. Biochem Pharmacol 79: 162-171, 2010 . 
4. Yu CC, Chiang PC, Lu PH, Kuo MT, Wen WC, Chen P and Guh JH: Antroquinonol, a natural ubiquinone derivative, induces a cross talk between apoptosis, autophagy and senescence in human pancreatic carcinoma cells. J Nutrition Biochem 23: 900-907, 2012.

5. Kumar VB, Yuan TC, Liou JW, Yang CJ, Sung PJ and Weng CF: Antroquinonol inhibits NSCLC proliferation by altering $\mathrm{PI} 3 \mathrm{~K} / \mathrm{mTOR}$ proteins and miRNA expression profiles. Mutation Res 707: 42-52, 2011.

6. Eisenhauer EA, Therasse P, Bogaerts J, et al: New response evaluation criteria in solid tumours: revised RECIST guideline (version 1.1). Eur J Cancer 45: 228-247, 2009.

7. Chen CC, Shiao YJ, Lin RD, Shao YY, Lai MN, Lin CC, Ng LT and Kuo YH: Neuroprotective diterpenes from the fruiting body of Antrodia camphorata. J Nat Prod 69: 689-691, 2006.

8. Lee TH, Lee CK, Tsou WL, Liu SY, Kuo MT and Wen WC: A new cytotoxic agent from solid-state fermented mycelium of Antrodia camphorata. Planta Med 73: 1412-1415, 2007.
9. Hseu YC, Chang WC, Hseu YT, Lee CY, Yech YJ, Chen PC and Yang HL: Protection of oxidative damage by aqueous extract from Antrodia camphorata mycelia in normal human erythrocytes. Life Sci 71: 469-482, 2002.

10. Chang JM, Lee YR, Hung LM, Liu SY, Kuo MT, Wen WC and Chen P: An extract of Antrodia camphorata mycelia attenuates the progression of nephritis in systemic lupus erythematosus-prone NZB/W F1 mice. Evid Based Complement Alternat Med 2011: 465894, 2011

11. Song TY and Yen GC: Protective effects of fermented filtrate from Antrodia camphorata in submerged culture against CCl4-induced hepatic toxicity in rats. J Agric Food Chem 51: 1571-1577, 2003 